\title{
ACUMULACIÓN DE CAPITAL Y “CRISIS DEL ESTADO DE BIENESTAR”. ELEMENTOS PARA LA COMPRENSIÓN DE SU DESARROLLO DURANTE EL SIGLO XX $X^{1}$
}

\section{CAPITAL'S ACCUMULATION AND "CRISIS OF THE WELFARE STATE". ELEMENTS FOR THE UNDERSTANDING OF ITS DEVELOPMENT DURING THE 20TH CENTURY}

\section{Yessenia Fallas Jiménez*}

RESUMEN

En el presente artículo se exponen alguna líneas generales para pensar la relación entre la crisis estructural del capital que viene desarrollándose desde finales de la década de 1970 y la crisis del llamado Estado de Bienestar. El artículo centra su atención en el análisis de las determinaciones históricas propias del origen de la relación entre ambos elementos para entender su desarrollo contradictorio hasta el presente.

PALABRAS CLAVE: ESTADO * BIENESTAR SOCIAL * CRISIS * CAPITAL * ECONOMÍA

\section{ABSTRACT}

This article attempts to present some general lines to examine the relationship between the structural crisis of capital that has been developing since the end of the 1970 s and the crisis of the so-called Welfare State. The article focuses on the analysis of the historical determinations of the origin of the relationship between both elements to understand its contradictory development up to the present.

KEYWORDS: STATE * SOCIAL WELFARE * CRISIS * CAPITAL * ECONOMY

$1 \quad$ El artículo es producto del proyecto de investigación denominado "Pry01-1902-2018-Fundamentos históricos de la génesis del Estado moderno y su relación con la producción capitalista” de la Escuela de Trabajo Social de la Universidad de Costa Rica. 


\section{INTRODUCCIÓN}

Para iniciar este diálogo, es necesario señalar algunos de los elementos básicos que sustentan este análisis, los cuales hacen referencia a un asunto propiamente metodológico que apunta a la forma en la que se hace el acercamiento al objeto de discusión en este artículo. En ese sentido, sin temor a reproducir un análisis "economicista" de este objeto, se parte del hecho que el mismo es, en el sentido marxiano, una síntesis de múltiples determinaciones y por tanto, "unidad en lo diverso" (Marx, 1989, p.51), lo que demanda que para entender la forma en la que se configuró la denominada crisis del Estado de Bienestar —objeto en este artículo-, sea necesario entender procesos históricos superiores que permiten aprehender las determinaciones propias de dicho objeto.

El primero de estos elementos refiere al hecho de que el capital es una relación social, que se funda en la manera en que los seres humanos establecen un intercambio con la naturaleza $y$, por lo tanto, un intercambio entre ellos mismos. Dicha relación social se caracteriza por una compulsión por el valor, cuyo fin según Marx:

es extraer trabajo excedente a través del alargamiento del tiempo de trabajo -es una relación de compulsión que no se apoya en ninguna relación personal de dominación $y$ dependencia, si no que surge simplemente de la diferencia en las funciones económicas, esta relacióncapital, como relación de compulsión, es común a [varios] modos de producción, pero el modo específicamente capitalista de producción también posee otros medios de extraer plusvalía (Marx, citado en Mészáros, 2002, p.708).

Es posible observar a lo largo del desarrollo de esta relación, una determinación histórica que se manifiesta en la forma de un imperativo estructural, el cual se caracteriza por una necesidad constante de expansión que es guiada por la acumulación. En palabras de Mészáros (2002), "el imperativo estructural expansionista del capital es la constante extracción de trabajo excedente de una forma o de otra, de acuerdo con los cambios en las circunstancias históricas" (p.120).

En segundo lugar, esta relación social requirió de un proceso histórico altamente complejo para su consolidación, pasando incluso de las llamadas formas precapitalistas de capital (Marx, citado en Mészáros, 2002), hasta llegar a su momento de pleno desarrollo, en el sentido de haber alcanzado las condiciones históricas en las que la fuerza de trabajo se convirtió en una mercancía para el trabajador, a partir de este momento se tiene como resultado el hecho de que la mercancía se convierte en la forma de todos los productos del trabajo (Marx, 1983). Es aquí cuando:

... todos los productos serán convertidos en mercancías... la mercancía como forma necesaria del producto, $y$ por tanto la alienación del producto como forma necesaria de su apropiación implica una división del trabajo social plenamente desarrollada, (...) solamente en la base de la producción capitalista, por tanto también en la división capitalista del trabajo en el interior de la fábrica, (...) todos los productos necesariamente asumen la forma mercancía y todos los productores son necesariamente productores de mercancía. Consecuentemente sólo con la llegada de la producción capitalista el valor de uso es por primera vez mediado genéricamente por el valor de cambio (Marx, citado en Mészáros, 2002, p. 707).

En tercer lugar y relacionado con lo anterior, el fundamento de esta relación, es decir, la razón que rige la producción en la sociedad capitalista, dejó de ser el conjunto de las necesidades humanas para pasar a ser la acumulación de capital. Así:

Las más variadas cualidades del valor de uso deben ser sometidas a cantidades determinadas de valor de cambio, antes de que puedan adquirir su legitimidad propia para ser producidas; $y$ deben constantemente probar su viabilidad- no en relación a las necesidades humanas cualitativamente diferentes, si no bajo los 
criterios estrictamente cuantitativos de intercambio de mercancías (Mészáros, 2002, p.946).

En cuarto lugar, a lo largo de su desarrollo histórico, esta relación logró convertirse en un sistema de control de las relaciones sociales que hizo parte de sí mismo a todas las relaciones existentes, pues literalmente la forma en la que el capital domina la producción le dio la vuelta al planeta durante el siglo $\mathrm{xx}$, realizando plenamente su tendencia estructural a la globalización impulsada por su imperativo de la expansión.

Según Mészáros (2002), la globalización es una tendencia que "emana" de la naturaleza del propio capital desde su comienzo y su significado real hoy adquiere la forma de un "sistema internacional de dominación y subordinación” (p.111). Para el autor, este proceso de envolvimiento de la totalidad de las relaciones sociales existentes en el planeta en la forma de una globalización, trae consigo una estela de desigualdades creciente para la gran mayoría de las personas, pues a pesar de presentarse como "muy benéfica, nada ofrece a los "países subdesarrollados" además de la perpetuación de la taza diferenciada de explotación" (p.64).

Al mismo tiempo, el proceso a partir del cual el capital subordinó todas los ámbitos de la vida en sociedad a su imperativo estructural de la expansión para la acumulación, abarcó desde los aspectos vinculados con la producción de mercancías lujosas hasta la producción de bienes considerados imprescindibles para la vida humana, relacionados a la satisfacción de las necesidades básicas como alimento, salud $y$ abrigo.

Una vez alcanzado el grado máximo de expansión extensiva, el capital abarcó todas las posibilidades de expansión intensiva: desde las formas más sofisticadas de reducción del tiempo de trabajo necesario para la producción de una determinada mercancía, hasta las formas más destructivas de completar su ciclo de realización, tales como, la obsolescencia programada. Esta situación acarrea no solamente formas cada vez más deshumanas de explotación de la fuerza de trabajo, sino también procesos altamente acelerados de destrucción de la naturaleza.

Una vez aclarados estos puntos de partida, se hace necesario identificar un elemento central para este análisis: dado que el capital alcanzó durante el siglo xx un alto grado de control sobre la totalidad de relaciones sociales en todos sus ámbitos, para entender las transformaciones del Estado y de la Política Social hoy (incluyendo la llamada crisis del Estado de Bienestar), se hace necesario entender las transformaciones en el proceso de acumulación de capital, como camino metodológico indispensable para analizar el desarrollo del Estado y la Política, como síntesis de múltiples determinaciones.

En ese sentido, dos grandes acontecimientos marcaran el proceso de acumulación de capital en el siglo xx, por un lado, la crisis de 1929 y, por otro, la crisis en el proceso de acumulación que se empieza a gestar a finales de la década de 1970.

\section{ACUMULACIÓN DE CAPITAL Y CRISIS DE 1929}

La crisis de 1929 se puede considerar de naturaleza cíclica en el sentido dado por Marx. La relación entre producción y consumo - radicalmente redefinida en el capitalismotrae consigo un alto espectro problemático, justamente porque la primera está fundamentada en la producción de mercancías y no de necesidades humanas. Esta relación altamente problemática entre ambos elementos, trae la necesidad de las crisis a lo largo del desarrollo histórico del capitalismo.

A través del desarrollo del capital, la relación entre las dimensiones de la producción, el consumo y la circulación tiende a fortalecerse y ampliarse, lo cual promueve motivaciones internas necesarias para la reproducción dinámica del capital en escala cada vez más amplia. Así, las crisis cíclicas y las limitaciones inmediatas de cada una de las dimensiones anteriores pueden ser superadas gracias a las interacciones que se puedan establecer entre estas (Mészáros, 2002). Gracias a esta interacción se superan las limitaciones de cada dimensión, por ejemplo, "la barrera inmediata para la producción es positivamente superada por la 
expansión del consumo y viceversa" (Mészáros, 2002, p. 798).

De esta forma es que los límites del capital parecen ser verdaderamente superados y no únicamente postergados y desplazados, junto con ello las contradicciones inmediatas (en la forma de crisis) pueden ser, al mismo tiempo, utilizadas como palancas para aumentar el aparentemente ilimitado poder de autoexpansión del capital. Mientras este tipo de mecanismo de autoexpansión (la regulación de la relación entre las tres dimensiones) que desplaza las contradicciones siga funcionando, no se presenta ninguna crisis de naturaleza estructural, a pesar de que se presentan crisis "cíclicas" de todas las formas, dimensiones y duraciones ${ }^{2}$.

Esto se observa en la crisis de 1929, que según Mészáros (2002), "fue una crisis de "realización”, debido al nivel marcadamente bajo de producción y consumo comparado con el periodo de posguerra" (p.798). Esta crisis:

... por más grande que fuera (...) estaba lejos de ser una crisis estructural, al dejar un gran número de opciones abiertas para la sobrevivencia continuada del capital, así como para su recuperación y su reconstitución más fuerte que nunca en una base económicamente más saludable y más amplia (Mészáros, 2002, p.793).

Por tal razón, la crisis de 1929 otorgó un estímulo para la reorganización de las fuerzas constituyentes del sistema económico posteriormente al límite productivo alcanzado a través de los avances productivos del periodo pos I Guerra Mundial y que no tenían cómo ser contenidos en las relaciones de poder existentes hasta el periodo inmediatamente anterior a la

2 Vale la pena mencionar el papel que el Estado moderno ocupa en términos de crear la "ayuda" externa que el capital necesita en determinados momentos de crisis. Este texto no tiene como objetivo analizar la relación entre capital y estado moderno, sobre ese asunto puede consultarse el documento "Estado moderno y capital: un análisis de las determinaciones históricas de su origen", presentado en la Jornada de Investigación de la Escuela de Trabajo Social, 2019. crisis; lo que permitiría dinamizar el proceso de acumulación, teniendo como resultado la revigorización de dicho proceso ahora bajo la hegemonía estadounidense.

Según Mészáros (2002), esta reconfiguración de las fuerzas constituyentes del capital significó:

$\diamond \quad$ El cambio del imperialismo multicentrado a un sistema de dominación global bajo la hegemonía de los Estados Unidos, sistema que se vuelve más dinámico y económicamente más viable e integrado.

$\diamond \quad$ El establecimiento del sistema monetario internacional y de otros órganos de regulación de las relaciones entre los capitales.

$\diamond \quad$ La exportación de capital a gran escala y "la repatriación" segura de las tazas de ganancia que no podrían ser producidas en los países de origen.

$\diamond \quad$ La incorporación relativa $y$ en diversos grados de las economías de las llamadas sociedades poscapitalistas en la propia estructura de intercambio capitalista.

Es importante el hecho de que sobre la base material de esa organización de las fuerzas constituyentes del capital, es que en el periodo pos crisis de 1929 (en el que se da una recuperación en el proceso de acumulación) se observó: a) una creciente intervención estatal, la cual contribuyó al proceso de expansión de capital; b) el tránsito de los sectores de la industria privada que quebraron durante la crisis hacia el sector público ( $y$ que prontamente se vieron en el contraflujo, es decir, hacia el sector privado, una vez que adquirieron nuevos niveles de eficiencia y recuperación en la producción de capital); c) un aumento de los índices de empleo (particularmente en el periodo de guerra bajo la forma del "pleno empleo") y d) una apertura de nuevos ramos de producción especialmente dirigidos al consumo de desperdicio (consumo de masas).

Vale la pena detenerse en el primer elemento, pues es justamente sobre este que algunas interpretaciones se basan para sustentar la idea de que, posterior a la crisis de 1929, 
la configuración del Estado moderno cambió radicalmente, cuya intervención alcanzó dimensiones sin precedentes tanto en cantidad (abarcando amplias espacios y dimensiones de la vida social desde la producción de mercancías hasta la ampliación de la política pública) como en formas (especialmente la política social referida a seguridad $y$ asistencia social $)^{3}$.

De la misma manera $y$ a pesar de que en Costa Rica, entre otras razones, por el carácter de economía periférica, no sea posible hablar de la existencia de un Estado de Bienestar, tal y como en los países del capitalismo central, se puede mencionar que sí hubo un crecimiento importante en las formas de intervención estatal acordes con la relación centro-periferia propias de una economía basada en las exportaciones.

Es así que se observa en las décadas contenidas entre 1940 y 1970, un aumento en la inversión del Estado en Política Pública y Social. Según Vega (1986), junto al fortalecimiento de la industria y la exportación, la política de los gobiernos socialdemócratas durante 1950-1969, se orientó a fortalecer la participación en el mercado de consumo de los sectores de clase media de la población nacional.

Además de la expansión del sector público durante las décadas de 1950 a 1970, el gasto público pasó de ser $33 \%$ en 1950 a 55,6\% en 1960 y 67,4\% en 1970, la cantidad de empleados públicos pasó de 35000 en 1960 a 100000 en 1975 y la inversión del Estado durante estos años permitió que la tasa de desempleo pasara de 7,3\% en 1973 a 4,6\% en 1978 (Vega, 1986).

3 Con respecto a la relación entre la reconfiguración de las fuerzas constituyentes del capital, el Estado de Bienestar (expresado comúnmente en el keynesianismo y las políticas de pleno empleo) y el complejo militar industrial, Mészáros ofrece un debate interesante, pues desde su punto de vista fue debido a la activación del complejo militar industrial en la II Guerra Mundial que se empezaron a constatar los resultados de las políticas keynesianas, ya mucho antes creadas y que sin la plataforma económica de la II Guerra Mundial no habrían conseguido mostrar sus resultados positivos, de la misma manera que las políticas de pleno empleo hallaron su realización en el marco de la II Guerra Mundial.
En términos de la inversión del Estado costarricense en política de vivienda, por ejemplo, esta pasó de 19171 obras construidas en el quinquenio de 1961-1965, a 19580 y 39169 en los quinquenios de 1966-1970 y 1971-1975, respectivamente (Bogan, 1979). Es importante mencionar que la inversión en vivienda varió significativamente de región a región, así por ejemplo, mientras que en el quinquenio 19611965, la inversión en el Valle Central permitió la construcción de 16005 viviendas (13 946 en aglomeraciones urbanas), en el Pacífico Central de 542, en el Pacífico Norte de 707, en el Pacífico Sur de 300, en la región Norte de 368 y en la Atlántica de 225. Estos datos evidencian las consecuencias de la migración interna y la dirección que, frente a esta, toma la política social (DGEC, 1963).

En cuanto al acceso a la seguridad social, en el año 1963, se estimó que del total de la población (1 333 432), el 97,8\% no tenía acceso a la seguridad social, mientras que el restante $2,3 \%$ sí lo tenía. De este último grupo, el $55,5 \%$, pertenecía a población inactiva y el restante a población activa, mayoritariamente ocupada (DGEC, 1963). En esta misma línea, Vega (1978) se refiere a ese contexto de la siguiente forma:

... lo que ha venido quedando definido desde ese entonces como proyecto histórico de cambio que ha respetado $y$ perfeccionado al esquema clásico de la democracia liberal - proyecto que no ha podido ser modificado por la oposición antiliberacionista - es el lanzamiento de la industrialización y en general del desarrollo económico sobre la base amplia de una banca y de un sector de economía estatizada, bajo un marco administrativo altamente burocratizado, de orientación paternalista, que en su aspecto "progresista" promueve la ampliación y movilidad de los sectores urbanos medios, estabiliza a los populares, $y$ se abre permanentemente a "negociar" con los empresarios locales y extranjeros de cuyo desarrollo se hace responsable (p. 125). 
Es importante resaltar el hecho de que la reconfiguración en los procesos de acumulación de capital, fue la base material para la expansión del Estado y las políticas públicas que caracterizaron las llamadas décadas gloriosas del capitalismo en los países centrales ${ }^{4}$.

\section{CRISIS ESTRUCTURAL DEL CAPITAL}

A finales de la década de 1970, el proceso de acumulación de capital experimentó uno de los momentos más determinantes de su desarrollo histórico. Contrario a las crisis anteriores cuya naturaleza estaba referida a la relación entre las dimensiones de producción, circulación y consumo, la crisis que se presentó $-y$ que continúa afectando el proceso de acumulación de capital- dejó ver que su naturaleza era totalmente diferente a la de las crisis anteriores, esta develaba límites vinculados a la propia estructura de la relación capital ${ }^{5}$.

Una crisis de carácter estructural afecta la totalidad de un complejo social, en todas las relaciones, en todas las partes constituyentes propias $y$ articuladas a dicho sistema, contrario a lo que sucede con las crisis cuya naturaleza no es estructural y que se restringen solamente a algunas de sus partes o dimensiones como se observó en el caso de la crisis de 1929 (y su naturaleza vinculada a la relación entre las tres dimensiones anteriormente mencionadas), una

$4 \quad$ Al respecto se sugiere ampliar en el apartado 18.2.5 del libro Más allá del capital. Rumbo a una teoría de la transición, de Itsván Mészáros. A pesar de tener varios inconvenientes en la traducción realizada en la edición en español (Vadell Hermanos, 2001), el análisis de los elementos colocados por el autor bien vale la pena el esfuerzo comparativo con la edición de la obra en inglés (Monthly Review Press, 1995) o portugués (Boitempo, 2002).

5 Vale aclarar que "la crisis estructural no se origina por sí sola en alguna región misteriosa: reside dentro y emana de las tres dimensiones internas anteriormente mencionadas. No obstante, las disfunciones de cada una, consideradas separadamente, deben ser distinguidas de la crisis fundamental del todo, que consiste en un bloqueo sistemático de las partes constituyentes vitales [del sistema]" (Mészáros, 2002, p. 798). crisis estructural coloca en riesgo incluso la "sobrevivencia continua de la estructural global" (Mészáros, 2002, p. 797).

En el caso de las crisis cíclicas, si la intervención a través de diferentes mecanismos permitía la continuada y sana acumulación - pues la misma demanda cambios solamente en las dimensiones afectadas - en la crisis estructural sucede todo lo contrario. Es justamente esto lo que caracteriza la crisis de finales del siglo $\mathrm{xx}$, esta es una crisis estructural y por tanto, de todo el sistema, por medio de la cual "entramos en una fase de inestabilidad sin precedentes” (p.735).

No se puede extender las explicaciones sobre la complejidad de esta crisis, pero se debe mencionar que su carácter refiere a la "activación de los límites absolutos" vinculados a las determinaciones estructurales del sistema del capital y que la misma "emana de la baja eficiencia $y$ de la asustadora insuficiencia de la extracción de trabajo excedente, con inmensas implicaciones para las perspectivas de sobrevivencia del propio sistema del capital" (Mészáros, 2001, p. 104). Al mismo tiempo, es necesario apuntar algunas características generales que según el autor, acompañan al desarrollo de esta crisis:

$\diamond \quad$ Su carácter es universal y no restringido a una esfera (financiera, comercial) o dimensión específica como la crisis de 1929.

$\diamond \quad$ Su alcance es realmente global y no limitado a un grupo de países.

$\diamond \quad$ La escala de tiempo en la que se configura es extensa, continua y permanente, no limitada y cíclica como fue el caso de las crisis anteriores.

$\diamond \quad$ Su modo es "rastrero" pues no se manifestó en modo de erupciones o colapsos como en el pasado (aunque no es posible excluir la posibilidad de esas erupciones), sino que se extiende de forma prolongada $y$ casi permanente.

Esta crisis se presenta también en un escenario en que el capital alcanzó un alto desarrollo de las fuerzas productivas que tiene 
dos consecuencias fundamentales, aunque no únicas, en el proceso de acumulación.

Por un lado, el acelerado desarrollo de las fuerzas productivas provocó un mayor nivel de eficiencia en la producción, lo que a su vez propició el establecimiento del desempleo crónico. Esto ha llevado a grandes contingentes de trabajadores a la deriva frente a un mercado de trabajo que no tiene condiciones para absorberlos como fuerza de trabajo; solo para mencionar un ejemplo, los datos de desempleo muestran que para el año 2017, la cantidad de personas desempleadas en el planeta era de 201 millones, cifra que aumentó para el año 2018 en 2,7 millones (Organización Internacional del Trabajo, 2017).

Por otro lado, dada su naturaleza, la crisis estructural no se limita a la esfera socioeconómica, sino que impacta todas las esferas de la vida en sociedad, desde la economía, pasando por la política hasta llegar a la propia subjetividad humana.

No es por casualidad que se haga frente cotidianamente a estadísticas que indican un aumento del deterioro de la salud mental ${ }^{6}$ expresado en las tazas de suicidios, depresión y consumo de fármacos, con los cuales las personas intentan subsistir a la desazón y falta de significado de la vida, que no consigue ser satisfecho ni siquiera a través del consumo de mercancías para la satisfacción de apetitos artificiales (hoy más que nunca realizado a través del crédito $^{7}$ ) menos aún a través de las

$6 \quad$ Al respecto, en el Semanario Universidad en su edición del 5 de junio de 2019, Molina informaba que la "tasa nacional de suicidio pasó de 6,4 en el 2017 a 7,2 por cada 100 mil personas en el 2018, en línea con una tendencia creciente desde los 80's y los intentos de suicidio crecieron un 65\%".

En el diario La Nación, en su edición del 16 de noviembre del 2018, Barquero alertaba que según datos del Ministerio de Economía, Industria y Comercio, hubo un crecimiento del endeudamiento particularmente con tarjetas de crédito, así por ejemplo, la cantidad de tarjetas de crédito que circulan en el país aumentó en un 14,5\% en un plazo de 12 meses, pasando de 2528051 tarjetas el 31 de julio de 2017 a 2896067 el 31 de julio de 2018. relaciones interpersonales ${ }^{8}$. Así, por ejemplo, según la OMS para el año 2018, 300 millones de personas en todo el mundo fueron afectadas por la depresión y cada año se suicidan alrededor de 800 millones de personas, siendo el suicidio la segunda causa de muerte de los jóvenes entre 25 a 29 años. Para esta misma organización, la depresión se debe a interacciones complejas de factores de carácter social, psicológico y biológico. Quienes han vivido situaciones consideradas como adversas (desempleo, luto, traumatismos psicológicos) tienen más posibilidades de sufrir cuadros de depresión (OMS, 2018).

En este escenario, las propias estructuras políticas en la forma de Estado moderno experimentan también las determinaciones inevitables de la crisis estructural del capital. Así:

... comprensiblemente la actual crisis estructural del capital afecta en profundidad todas las instituciones del estado $y$ los métodos organizacionales correspondientes. Junto con esta crisis viene la crisis política en general, bajo todos sus aspectos, $y$ no solamente bajo los directamente preocupados con la legitimación ideológica de cualquier sistema particular de Estado (Mészáros, 2002, p. 106).

\section{NEOLIBERALISMO COMO PRETENDIDA SALIDA A LA CRISIS ESTRUCTURAL}

Una vez instalada la crisis, una serie de estrategias fueron diseñadas y ejecutadas como tentativas de superación; sin embargo, la naturaleza de dicha crisis, las salidas que antes tuvieron efectos positivos, actualmente -40 años después - no muestran resultados esperanzadores,

8 Es importante señalar las formas de violencia social expresadas individualmente (en la competencia por asegurar un empleo de cara al creciente desempleo crónico) y socialmente en la violencia reproducida a lo interno de las clases sociales y por parte del Estado, expresadas en la disputa cotidiana por la sobrevivencia (con actividades incluso consideradas como ilícitas) y la criminalización de la protesta y el movimiento social respectivamente. 
pues no es posible ni siquiera afirmar que en términos de la intervención del Estado y la gestión de la Política Pública y Social se regresara a los niveles alcanzados en las décadas gloriosas del capitalismo en Occidente. Como elemento inicial es fundamental entender algunos asuntos vinculados al neoliberalismo.

El primero de ellos refiere al hecho de que más que una pretendida forma ideológica o mero discurso político, el neoliberalismo aparece como forma de pensar y gestionar salidas a la crisis que privilegian el mercado como espacio "regulador" por excelencia de las relaciones sociales.

En segundo lugar, como parte de esta pretendida salida a la crisis, todos los negocios rentables en términos de la producción de capital que en las décadas pasadas estaban en las manos del Estado, debían ser trasladados a manos de empresas privadas, cuya eficiencia productiva es superior. Fue así como negocios vinculados a electricidad, telecomunicaciones, minería, salud, entre otros, fueron trasladados a manos privadas bajo las más diversas figuras legales como la venta de empresas, la apertura comercial de los monopolios (que en varios casos dejó pérdidas millonarias para las pequeñas empresas estatales que competían con gigantes privados) y ya en la década de los 90, la tercerización y las concesiones de bienes o servicios para ser administrados por las empresas privadas.

Así, por ejemplo, según Vidal (2001) para finales de la década de 1990, más de dos terceras partes de los países de América Latina habían privatizado las empresas de telecomunicaciones. América Latina:

... obtuvo ingresos por un valor de US\$59.000 millones como consecuencia de la venta de 694 empresas durante el periodo 1990-1994 equivalentes a más de la mitad de los US\$104.000 millones obtenidos por ese concepto por todos los países en desarrollo. Si consideramos el periodo de 1990 a 1996 las privatizaciones implicaron ingresos por 82.417 millones de dólares que representan el $53 \%$ de los ingresos totales entre los llamados mercados emergentes (p.11).
Este proceso de "apertura comercial" de los monopolios estatales $y$ venta de las empresas del Estado, fue legitimado entre otros elementos por el discurso de la eficiencia. A este discurso le faltó explicar cómo en los años venideros, las empresas privadas no serían eficientes para la gestión de las declaratorias de quiebra y las crisis cíclicas presentadas a principios del siglo XXI (2002-2008). Por ejemplo, en el año 2008, la Reserva Federal de los Estados Unidos rescató de la bancarrota a una de las mayores aseguradoras del planeta, American International Group (AIG) al inyectar 85000 millones de dólares para impedir su derrumbe y a cambio de ello se hizo del $79,9 \%$ del capital de la sociedad 9 .

En ese mismo año, la compañía General Motors recibió a través de su brazo financiero (GMAC) la suma de 5000 millones de dólares por parte del Departamento del Tesoros de los Estados Unidos. Al respecto, el diario español El Mundo anunciaba que la ayuda consistiría en "una inversión directa del Gobierno a cambio de una participación accionaria preferencial y en los dividendos".

Ese discurso tampoco logró explicar por qué las instituciones poco o nada rentables en la producción de capital, no eran apetecidas por las empresas privadas cuando a partir de 1980, se dio el proceso de venta de bienes que estaban en manos del Estado, por ejemplo, en el caso de Costa Rica se pueden mencionar las instituciones de asistencia social, tales como, el PANI, el IMAS, entre otras ${ }^{10}$.

$9 \quad$ Así anunciaba el diario El País, la astronómica operación financiera transcribiendo parte del comunicado general de la Reserva Federal que afirmaba que en aquellas circunstancias, "un colapso [fracaso desordenado, en el literal] de AIG elevaría los ya significativos niveles de fragilidad del mercado financiero" (Pozzi, 17/09/2008).

10 A pesar de esto no se debe ignorar el hecho de que en estas instituciones se empezó a desencadenar, a finales del siglo xx, un proceso de tercerización de los servicios, a través de la contratación de profesionales liberales para la ejecución de algunos de sus programas y proyectos (procesos como los de evaluación y valoración para adopciones en el caso de PANI o Programas como Construyendo Oportunidades en el IMAS, ejecutado entre 1999 y 2001). 
En tercer lugar, un elemento que caracterizó la intervención estatal a partir de la década de 1990, fue la tendencia progresiva a disminuirla a través de la política pública $y$ particularmente de la política social, dirigida anteriormente a la población de los estratos con ingresos más bajos y medios de la población. Así, a partir de 1980, la política social sufrió un proceso de focalización que se vio acompañado de formas de gestión inexistentes en el pasado, como la tercerización de los servicios, estrategia de gestión que se mostró más eficiente y austera, pues implicó una disminución de los gastos del Estado en contrataciones y costos derivados de las mismas: vacaciones, seguro de salud, incapacidades, aguinaldos, etc.

Paralelamente a este proceso de reducción y focalización de la intervención del Estado, su presencia se mantuvo por la vía del parlamento o incluso, por la del mismo poder ejecutivo, siempre que contribuyera al buen funcionamiento del proceso de acumulación: inversión económica en sistemas y en vías de transporte para la circulación de mercancías, prohibiciones a los monopolios o legalización de los mismos dependiendo de las necesidades coyunturales del proceso de acumulación, aprobación de tratados de libre comercio que permitían la circulación de mercancías en escala global con menores obstáculos, entre otros.

En síntesis es posible observar que el agotamiento de un patrón interventivo estatal se desarrolla progresivamente como parte de un agotamiento del periodo de ascensión en el proceso de acumulación de capital, propios del periodo pos II Guerra Mundial ${ }^{11}$.

Se puede observar al mismo tiempo que el "patrón interventivo" cuya contribución al proceso de acumulación es innegable posterior a la

11 Debe aclararse aquí que en el "periodo neoliberal" se observan otras estrategias de superación de la crisis como, por ejemplo, el acelerado proceso de financierización de la economía -incluida la política social- (al respecto puede observarse un análisis crítico en Chesnais, 2009), la obsolescencia programada y la producción del desperdicio, así como, el desarrollo del Complejo Militar Industrial (Mészáros, 2002), estrategias que hasta ahora no se mostraron efectivas en la superación de la crisis estructural. crisis de 1929, transita en las última décadas del siglo xx hacia formas de presencia estatal -intervenciones estatales - cuyo carácter se vincula más al control y la punición, tanto en la forma en que se ejecutan las políticas sociales ${ }^{12}$ como en la que se militariza la política de atención a la pobreza y se castiga el movimiento social ${ }^{13}$.

\section{CRISIS, NEOLIBERALISMO Y ESTADO DE BIENESTAR}

Si anteriormente se parte del hecho de que la base material propia de una bonanza en el proceso de acumulación fue la base del éxito de las políticas interventivas del Estado en Occidente durante las llamadas décadas gloriosas del capitalismo - a tal punto de ser considerado como un Estado de Bienestar en los países centrales-, cabe preguntarse sobre cuáles son las posibilidades de un retorno saludable y sustentable a aquel patrón de acción estatal en el actual momento de crisis estructural.

De igual forma, si se entiende que el Estado de Bienestar se apoyó sobre una economía relativamente saludable propia de un momento de bonanza en el proceso de expansión y acumulación de capital, cabría preguntarse también si en el momento de la crisis estructural es posible observar la existencia de esa base material o al menos una que muestre la estabilidad propia de las décadas anteriores a 1970, sobre todo si se considera con detenimiento que se atraviesa un momento histórico en el que los niveles de pobreza están en constante aumento, aunado a las tazas de desempleo y las formas de intervención de los Estados nacionales frente a la precarización de la vida cotidiana, cada vez más focalizadas y precarizadas ${ }^{14}$.

12 Puede profundizarse sobre este tema a través del texto de Fallas y Molina (2009). Transferencias monetarias condicionadas en Costa Rica: el caso del programa AVANCEMOS.

13 Al respecto, Jose Paulo Netto ofrece un interesante análisis en su artículo "Capitalismo y barbarie contemporánea" (2012).

14 Para analizar algunas determinaciones históricas, así como datos sobre pobreza y política social, se sugiere un acercamiento al texto "Política Social no capitalismo tardío" (Rosseti, 2009). 
Esta interrogante vale no solamente para los países periféricos, sino también para los países centrales otrora reconocidos por su "Estado de Bienestar". Ken Loach ofrece un vistazo dramático a la degradación de la intervención estatal en el centro capitalista en su película "Yo, Daniel Blake" (2016), en cuyo guión se expresa la angustia de la clase trabajadora que disfrutó de la expansión del Estado en las décadas anteriores y ve en el siglo XXI la precarización de su propia vida y junto con ello de la atención estatal.

En ese sentido, la naturaleza de la crisis hace que sea imposible reproducir — mucho menos universalizar - la expansión de "las conquistas" y concesiones del Estado de las décadas gloriosas del capitalismo en el siglo xx. Pero también hace que sea cada vez más difícil sostener las que fueron alcanzadas en el pasado, pues con la entrada del capital en una crisis estructural esas conquistas mostraron no ser sustentables a largo plazo. Como ejemplo, se observa en Costa Rica, las transformaciones en los sistemas de pensiones y las negociaciones cada vez más embarazosas de las convenciones colectivas de los sindicatos de trabajadores en los últimos años ${ }^{15}$. En ese sentido:

... el desaparecimiento ignomioso del Estado de bienestar social expresa claramente la aceptación del hecho de que la crisis estructural de todas las instituciones políticas ya viene fermentándose bajo la costra de la "política del consenso" hace más de dos décadas [escrito en 1995]. Lo que necesita ser subrayado aquí es que las contradicciones subyacentes de ningún modo se disipan en la

$15 \quad$ En México, por ejemplo, la modificación a "Ley del Instituto de Seguridad Social y Servicios Sociales de los Trabajadores del Estado" de 2007, aumentó la edad para jubilarse de 55 años a 60 años a ser alcanzada en el 2018. En Portugal, en 2013 se modificó la "Ley de pensiones" que aumentó la edad para jubilarse en un año, pasando de 64 a 65 años y estableciendo además que el aumento en la edad para la jubilación debe estar de acuerdo con el aumento de la esperanza de vida, con lo que pasó de 66 años y dos meses en 2016 a 67 años en 2019 (Fallas, 2006). crisis de las instituciones políticas, al contrario, afectan toda la sociedad de un modo nunca antes experimentado. Realmente, la crisis estructural del capital se revela como una verdadera crisis de dominación en general (Mészáros, 2002, p.800).

Inclusive, debido a la naturaleza de la crisis que se experimenta en la actualidad:

... a pesar de que una alteración coyuntural pudiese traer de vuelta, por lo menos por algún tiempo, un intento de institución de alguna forma keynesiana de administración financiera del Estado solamente podría existir por un periodo corto, dada la falta de condiciones materiales para facilitar su extensión por un periodo mayor, incluso en los países capitalistas dominantes (p. 26).

Es por esa razón que ganancias, inclusive defensivas y no contra-capitalistas como las que se experimentaron en el pasado $-y$ que estuvieron siempre dentro de los límites del propio proceso expansivo del capital- no tienen más viabilidad histórica. Valdría la pena entonces preguntarse acerca de cuáles deberían ser las finalidades $y$ horizontes de lucha de los movimientos que se asumen como movimientos contra el capital y cuyo espectro es cada vez más amplio: pasando por los movimientos de "causa única" hasta llegar a aquellos cuya naturaleza es declaradamente socialista.

En ese sentido, los desafíos que enfrenta la clase trabajadora parecieran apuntar a un horizonte mayor que el retorno al Estado de Bienestar (si es que en Costa Rica se puede mantener la afirmación de su existencia con todas las características que esto implica) y la expansión (o incluso la pretendida universalización) de las formas de intervención del Estado a través de la política pública, pues estas no fueron solamente acciones en el campo político, sino más bien formas de intervención estatal orgánicamente articuladas al estadio de desarrollo del capital y de su "saludable" proceso de expansión. 
Por tanto, la crisis estructural del capital es una oportunidad para profundizar la comprensión acerca de la naturaleza de la relación capital en el sentido de sus determinaciones estructurales más profundas ya apuntadas por el propio Marx en el siglo XIX, pero también para repensar el carácter de aquellas luchas cuyo horizonte se restringe a los límites deshumanos de la relación capital. Con lo anterior se apunta a la reorganización de los procesos productivos, la distribución de la riqueza, la universalización de los servicios públicos y la intervención estatal, pues las condiciones actuales muestran la imposibilidad de realización de dichos horizontes $y$ aunque en el mejor de los escenarios esto fuera posible, cabría preguntarse cuánto de ello contribuiría a una lucha genuina contra el capital por la emancipación humana.

\section{REFERENCIAS}

Barquero, M. (16 de noviembre de 2018). Deudas de tarjeta de crédito en Costa Rica aumentó \$166.000 millones en un año. La Nación. Recuperado de https://www.nacion.com/economia/indicadores/deuda-en-tarjetasde-credito-en-costa-rica-aumento/ TIFYFE04BVCOZII6ASE7VQQ57E/story/

Bogan, M. (1979). Vivienda y población en Costa Rica proyección: necesidades de vivienda hasta el año 2000. Memoria del Semanario Nacional de Demografía. Costa Rica. UCR.

Chesnais, F. (2009). La preeminencia de las finanzas en el seno del "capital en general", el capital ficticio y el movimiento contemporáneo de mundialización del capital. En Las finanzas capitalistas. Para comprender la crisis mundial, (pp. 79-150). Buenos Aires: Ediciones Herramienta.

Dirección General de Censos y Estadísticas. (1963). Censo de 1963: población y vivienda. Resultados provisionales obtenidos por muestreo. San José.

EFE. (30 de diciembre de 2008). La institución financiera de General Motors recibirá una ayuda de 5.000 millones de dólares. El mundo.es. Recuperado de https://www.elmundo.es/mundodinero/2008/12/30/economia/1230607781. html

Fallas, Y. (2016). Parlamento, Estado y capital en la óptica de Mészáros: un análisis de la obra Más allá del capital. (Tesis de doctorado sin publicar). Universidad Federal de Río de Janeiro, Río de Janeiro, Brasil.

Fallas, Y. y Molina, L. (2009). Transferencias monetarias condicionadas en Costa Rica: el caso del programa avancemos. Revista de Políticas Públicas, 13 (2), 219-229.

Lessa, S. (2013). Capital e Estado de Bem-estar. O caráter de classe das políticas públicas. São Paulo: Instituto Lukács.

Marx. K. (1983). El capital. Buenos Aires: Siglo XXI.

Marx. K. (1989). Introducción general a la crítica de la economía política/1857. Buenos Aires: Siglo XXI.

Mészáros, I. (2001). Más allá del capital rumbo a una teoría de la transición. Caracas: Hermanos Vadell.

Mészáros, I. (2002). Para além do capital. Rumo a uma teoría de transição. São Paulo: Boitempo.

Molina, L. (05 de junio de 2019). Los suicidios aumentan en Costa Rica ocultos entre las comunidades. Semanario Universidad. Recuperado de https://semanariouniversidad.com/especiales/suicidios-aumentan-en-costa-rica/

Netto, J. (enero-junio, 2012). Capitalismo e barbárie contemporânea. Argumentum, 4 (1), 202-222. Recuperado de http://www. abepss.org.br/arquivos/anexos/netto-josepaulo-201608060404028661510.pdf

O`Brien, R. (productora) y Loach, K. (director). (2016). Yo, Daniel Blake [cinta cinematográfica]. Reino Unido: Sixteen Films, Why not Productions y Wild Bunch.

OIT. (2017). Informe Perspectivas sociales y del empleo en el mundo-Tendencias 2017. Ginebra. Recuperado de https://www. ilo.org/global/research/global-reports/ weso/2017/lang--es/index.htm

Organización Mundial de la Salud-oms. (2018). Depresión. Datos y cifras 2018. 
Recuperado de https://www.who.int/es/ news-room/fact-sheets/detail/depression

Pozzi, S. (17 de setiembre de 2018). EE. UU acude al rescate de AIG para evitar una nueva quiebra. El país. Recuperado de https:// elpais.com/diario/2008/09/17/economia/ 1221602401_850215.html

Rosetti, E. (2009). Política Social no capitalismo tardío. São Paulo: CORTEz.

Vidal, G. (2001). Privatizaciones, fusiones y adquisiciones: las grandes empresas en América Latina. México D.F: ANTROPHOS.
Vega, J. L. (1978). Sociedad, Estado y Política en la Costa Rica de hoy. Ponencia presentada en el III Congreso Centroamericano de Sociología, Honduras.

Vega, J. L. (1986). Hacia una interpretación del desarrollo costarricense: ensayo sociológico. San José: Editorial PRovenIR.

Fecha de ingreso: 21/06/2019 Fecha de aprobación: 15/10/2019 